\title{
Das Regieren von Emotionen in Räumen des betreuten Wohnens
}

\author{
N. Marquardt \\ Institut für Humangeographie, Goethe-Universität Frankfurt am Main, Theodor W. Adorno-Platz 6, \\ 60629 Frankfurt am Main, Germany \\ Correspondence to: N. Marquardt (n.marquardt@em.uni-frankfurt.de)
}

Received: 04 August 2014 - Revised: 17 May 2015 - Accepted: 08 June 2015 - Published: 02 July 2015

\begin{abstract}
Kurzfassung. Der Aufsatz schlägt die Verbindung und Erweiterung von Analysen des (neoliberalen) Regierens mit nicht-subjektzentrierten und affekttheoretischen Ansätzen vor. Anhand einer Analyse des sozialpolitischen und sozialarbeiterischen Umgangs mit Wohnungslosen wird nachvollzogen, welcher Gewinn sich aus der Verbindung von gouvernementalen und affekttheoretischen Perspektiven ergeben kann. Aus einer gouvernementalen Perspektive wird zunächst nachgezeichnet, wie Affekte und Emotionen in Räumen des betreuten Wohnens für Wohnungslose zum Gegenstand fürsorglicher Intervention werden. Im betreuten Wohnen kommen Mikrotechniken zum Einsatz, die auf eine ,ausgewogene“ emotionale Bindung an Wohnräume und ihr Inventar hinarbeiten. Das betreute Wohnen ist von Problematisierungen durchzogen, die Wohnungslosigkeit als emotionale Haltung der Rastlosigkeit und Unruhe, als einen Mangel an Verbundenheit mit Orten und Dingen deuten. Gleichzeitig wird den Untergebrachten häufig auch eine übersteigerte affektive Bindung an Dinge unterstellt, die sogenannte „Horder“ und „Messies“ an einer sozial unauffälligen Haushaltsführung hindere. Eine gouvernementale Analyse kann die therapeutische Rationalität sichtbar machen, die diesen Problematisierungen zugrunde liegt. Eine gouvernementale Analyse allein bietet gleichwohl keine Möglichkeit, alternative Erzählungen über die Bedeutung affektiver Beziehungen für das Wohnen zu entwickeln. Mithilfe unterschiedlicher affekttheoretischer Ansätze geht der Aufsatz daher auch der Frage nach, wie sich jenseits therapeutisierender Perspektiven über das Wohnen und die Bedeutung von Bindungen an Orte und Dinge nachdenken lässt. Nicht-subjektzentrierte Konzepte von Affektivität ermöglichen solche alternativen Erzählungen und eröffnen neue Fluchtlinien der Kritik: Wohnen wir sichtbar als immer schon „betreut“, eingelassen in ein Netz von intersubjektiven und interobjektiven Beziehungen.
\end{abstract}

\section{Einleitung}

Dieser Aufsatz geht der Frage nach, wie Wohnungslose in Räumen des betreuten Wohnens zu Objekten des „Regierens" im Sinne Foucaults werden. Damit stellt der Aufsatz die Frage nach der Ausübung gesellschaftlicher Macht durch sozialbehördliche und sozialarbeiterische Praktiken der Versorgung und Betreuung von vermeintlich abweichendem Verhalten. Zwei Theoriefelder sollen im Rahmen der Diskussion zusammengeführt werden: zum einen die Foucault'sche Theorie der Gouvernmentalität, zum anderen unterschiedliche Philosophien des Wohnens, denen gemeinsam ist, dass sie die Bedeutsamkeit von Affekten und Gefühlen für das Wohnen in den Mittelpunkt des Interesses stellen.
Spätestens seit dem Beginn der studies in governmentality, die sich zunächst im angelsächsischen Sprachraum (Barry et al., 1996) und wenig später auch in Deutschland etabliert haben (Lemke et al., 2000), gehen Analysen neoliberaler Regierungsweisen den subjektivierenden Effekten nach, die gegenwärtige Formen des Regierens zeitigen. Sie zeichnen nach, an welchen Selbstentwürfen und Formen der ,,inneren Führung" wir uns orientieren sollen und wie wir angerufen werden, uns selbst zu optimieren (Rose, 1998, 1999; Bröckling, 2007, 2013). Folgt man den vorliegenden Analysen, dann scheint die Arbeit an einem rational-kalkulierenden Selbstverhältnis die wichtigste Handlungsvorgabe und Zielstellung gegenwärtiger Regierungsweisen zu sein: Es gilt, sich selbst 
unternehmerisch und ökonomisch vorausschauend zu verhalten und Risiken vernünftig zu kalkulieren, um dann flexibel und kreativ mit ihnen umgehen zu können.

Hinter den Forderungen nach einem rational kalkulierenden Selbstmanagement verbirgt sich allerdings auch ein umfassender Anspruch an die Arbeit an der eigenen emotionalen Disposition und damit auch eine affektive Dimension sozialer Ordnung. Prozesse der Subjektivierung umfassen notwendig auch Versuche der Erzeugung und Justierung von Gefühlen. Die Aufgabe etwa, lebenslang flexibel zu bleiben und immer neue Wege zu finden, gesellschaftliche Risiken im eigenen Leben kreativ abzufangen, impliziert nicht nur den Aufruf zu rationalem Handeln, sondern ebenso auch zur Kultivierung emotionaler Fähigkeiten. Um dieses Management von Gefühlen soll es im Folgenden gehen. In vielen gegenwartsbezogenen Studien zum Verhältnis von Regieren und Subjektivierung spielt das Regieren von Gefühlen bislang keine sonderlich prominente Rolle. Zwar zielt die Kritik an neoliberalen Subjektivierungsweisen darauf ab, gesellschaftliche Zumutungen immer auch als psychisch-emotionale $\mathrm{Zu}$ mutungen sichtbar zu machen. Gefühle und ihre Zurichtung sind häufig irgendwie „,mit gemeint“, wenn es um die kritische Auseinandersetzung mit gegenwärtigem Regieren geht. Wie aber Affekte zur Zielscheibe von politischen Rationalitäten werden und welche Techniken zur Regulation von Gefühlen zum Einsatz kommen, ist selten ein explizit ins Zentrum gerückter Gegenstand des Interesses. Gefühle taugen einem Großteil gouvernementaler Studien bestenfalls als „Verzierungen“ analytischer Narrative, gelten damit gleichsam aber auch als „Ablenkung“ von der wahren „Realpolitik“ des Regierens, wie Ann Stoler (2008:6) treffend feststellt. Folgt man Rosi Braidotti (2007), dann machen sich die governmentality studies eines ,residual Kantianism“ schuldig, indem sie implizit davon ausgehen, dass sich menschliches Handeln tatsächlich primär an rationalen Erwägungen ausrichte und deshalb für die Anrufungen des Regierens empfänglich sei. Damit reproduzieren sie letztlich eine von Regierungspraktiken selbst zugrundegelegte Konzeption von Subjektivität, die davon ausgeht, dass Subjekte als rationale Wesen sich verändern und „bessern“, wenn man nur angemessen an sie appelliert.

Um die Regulation von Gefühlen zum Gegenstand der Untersuchung zu machen, schlägt der Aufsatz eine Verbindung von gouvernementalen Konzepten des Regierens mit affekttheoretischen und nicht-subjektzentrierten Ansätzen vor. Anhand einer Analyse des Umgangs mit Wohnungslosen in Räumen des „betreuten Wohnens“ wird nachvollzogen, welcher Gewinn sich aus dieser Verbindung ergeben kann. Räume des betreuten Wohnens sind ein wichtiger Schauplatz der gesellschaftlichen Bearbeitung von Wohnungslosigkeit, die die sogenannte „Wohnfähigkeit“" von Wohnungslosen zum zentralen Problem erklärt. Um Wohnfähigkeit zu steigern, leitet die Wohnungslosenhilfe ihre Klient_innen im betreuten Wohnen nicht nur zu einer vernünftig kalkulierenden Haushaltsführung an. Wie zu zeigen sein wird, ist sie im- mer auch um die Modellierung angemessener Gefühlszustände als Voraussetzung für gesellschaftliche Reintegration bemüht. In dieser Problematisierung der „Psyche“ der Wohnungslosen finden sich Motive, die als idealtypisch für die gegenwärtige gesellschaftliche Regulation von Gefühlen gelten können. Reintegrative Hilfen für Wohnungslose machen auf besondere Weise sichtbar, ,,auf welche Weise die Subjekte über ihr Wohnen gefühlsmäßig vergesellschaftet werden“" sollen (Hasse, 2012:476, Betonung i.O.).

Aus einer gouvernementalen Perspektive zeichnet der erste Teil des Aufsatzes anhand von Interviews mit Expert_innen der Wohnungslosenhilfe und ethnographischen Aufzeichnungen zunächst nach, wie Affekte und Emotionen in Räumen des betreuten Wohnens zum Gegenstand von Beobachtung und fürsorglicher Intervention werden. ${ }^{1}$ Einerseits zeigt sich die Wohnungslosenhilfe traditionell von Problematisierungen durchzogen, die Wohnungslosigkeit als eine emotionale Haltung der Rastlosigkeit, eine mangelnde Verbundenheit mit Orten und Dingen deuten. Zum anderen wird den Untergebrachten immer wieder auch eine übersteigerte affektive Bindung an Dinge unterstellt, die sogenannte „Horder“ und „Messies“ an einer sozial unauffälligen Haushaltsführung hindere. Entsprechend ist das betreute Wohnen ein Ort des Einsatzes von Mikrotechniken, die beständig auf eine ausgewogene emotionale Bindung ihrer Klient_innen an Wohnräume, Inventar, Haustiere und andere Menschen hinarbeiten. Eine Analyse im Anschluss an Foucaults Konzept der Gouvernementalität kann die therapeutische Rationalität sichtbar machen, die dieser Hilfepraxis zugrunde liegt.

Eine gouvernementale Analyse allein bietet gleichwohl keine Möglichkeit, alternative Erzählungen über das Wohnen zu entwickeln. Die stillen Grundannahmen über Subjektivität und Wohnen, die die Betreuung wohnungsloser Subjekte im betreuten Wohnen anleiten, werden deshalb im zweiten Teil des Aufsatzes in ein Spannungsverhältnis zu unterschiedlichen Philosophien des Wohnens gestellt, die Wohnen als etwas anderes entwerfen, als es in der Praxis der behördlichen Hilfe für Wohnungslose vorausgesetzt wird. Die Diskussion unterschiedlicher, nicht-subjektzentrierter und affekttheoretischer Theorieperspektiven, die den Raum des Wohnens als Spiegel existenziell und praktisch gelebter Verhältnisse zu Welt und Selbst betonen, lässt aufscheinen, was aus phi-

\footnotetext{
${ }^{1}$ Das verwendete Interviewmaterial setzt sich aus Expert_inneninterviews mit Vertreter_innen der Wohnungslosenhilfe sowie aus ethnographischen Aufzeichnungen von teilnehmenden Beobachtungen bei Expert_innenrunden und Fachtagungen zusammen. Über die Expert_inneninterviews mit Vertreter_innen der kommunalen und freiverbandlichen Wohnungslosenhilfe sowie über die ethnographischen Beobachtungen konnten detaillierte Einblicke in die Hilfepraxis und die in der alltäglichen Arbeit wirksamen Problematisierungen erhoben werden. Ein besonderer Fokus lag bei den Interviews auf Trägern der freiverbandlichen Wohnungslosenhilfe, die in Einzelprojekten nach $\$ 67 \mathrm{ff}$. des Sozialgesetzbuches XII persönliche Hilfen in Form des betreuten Wohnens anbieten. Das Material wird anonymisiert verwendet.
} 
losophischer Sicht unter Wohnen verstanden werden kann. Ziel des Aufsatzes ist es nicht, aus diesen heterogenen Ansätzen eine einheitliche Theorie des Wohnens zu entwickeln, oder die Hilfepraxis des betreuten Wohnens zu diskreditieren. Was mithilfe der herangezogenen Ansätze aber der Kritik zugänglich gemacht werden kann, sind die machtvollen gesellschaftlichen und behördlichen Annahmen über Wohnen, die in der Hilfepraxis Geltung beanspruchen. Gleichzeitig ermöglichen die versammelten Ansätze alternative Erzählungen über das Wohnen und eröffnen so Fluchtlinien der Kritik als auch der potenziellen (Selbst)Ermächtigung. Wohnen wird sichtbar als immer schon „betreut“, eingelassen in ein Netz von intersubjektiven und interobjektiven Beziehungen.

\section{Wohnen trainieren: Problematisierungen von Wohnfähigkeit in der Wohnungslosenhilfe}

Räume des betreuten Wohnens sind in Deutschland seit den 1980er Jahren zentraler Bestandteil der auf gesellschaftliche Reintegration ausgerichteten Hilfe für Wohnungslose. Ähnlich wie die Prävention von Wohnraumverlust (etwa durch Mietschuldenübernahme) und wohnungspolitische Instrumente, die den Wiedereinstieg in den Wohnungsmarkt erleichtern sollen (etwa über Vereinbarungen der Kommunen mit Wohnungsbaugesellschaften), ist auch das betreute Wohnen Ergebnis einer weitreichenden Reform des Hilfesystems in den 1980er Jahren. Insbesondere reagiert es auf die Kritik an den potenziell stigmatisierenden Effekten großer Notunterkünfte. $^{2}$

Anleitende Idee des betreuten Wohnens ist, dass eine gesellschaftliche Reintegration von Wohnungslosen vor allem dann gelingt, wenn diese nach dem Verlust ihrer Wohnung weiterhin möglichst „,normale Wohnsituationen“ erleben. Gleichzeitig ist das betreute Wohnen geprägt von der Vorstellung, dass es wichtig ist, die „Wohnfähigkeit“ von Wohnungslosen zu stärken, bevor sie erfolgreich in den Wohnungsmarkt vermittelt werden können. Während die Hilfepraxis also einerseits auf eine schnelle Unterbringung von Wohnungslosen abzielt, um Straßenobdachlosigkeit zu ver-

\footnotetext{
${ }^{2}$ Bei betreuten Wohnräumen handelt es sich in der Regel um von Trägern der Wohnungslosenhilfe angemietete Projektwohnungen, die häufig dezentral über den Stadtraum verteilt sind, sowie um sogenannte Übergangshäuser mit mehreren Projektwohnungen. Anders als große Notübernachtungsstätten fallen diese Wohnungen kaum öffentlich als Wohnungslosenunterkünfte auf. Die Betreuung wird in der Regel nach \$67ff. des Sozialgesetzbuches XII erbracht. Gemäß der etablierten Arbeitsteilung zwischen den Kommunen und der freiverbandlichen Wohnungslosenhilfe (getragen von Wohlfahrtsverbänden wie Caritas, Diakonie etc.) geben im Fall des betreuten Wohnens die Kommunen (Kostenträger) die Aufgabe der Hilfen für Wohnungslose (Leistungsempfänger) an die Einrichtungen der freiverbandlichen Wohnungslosenhilfe (Leistungserbringer) weiter. Für eine detaillierte Darstellung der verwaltungsorganisatorischen Grundlagen der Hilfe vgl. Specht (2013).
}

meiden, etabliert sie andererseits eine der Reintegration in reguläre Mietverhältnisse vorausgehende Zwischenphase in Räumen der Hilfe. Das betreute Wohnen ermöglicht intensive soziale Beziehungen zwischen Wohnungslosen und Mitarbeiter_innen der Wohnungslosenhilfe. Die Betreuung wird als Angebot für die Betroffenen verstanden, sich in „kleinen Schritten“ wieder an das Wohnen zu „gewöhnen“, ohne von der Autonomie eigenverantwortlicher Haushaltsführung „gleich wieder überfordert“ zu sein.

Dem Konzept der „kleinen Schritte“ entsprechen im Alltag viele kleinteilige (und kleinliche) Maßnahmen, die die Wohnfähigkeit der Klient_innen verbessern sollen, indem sie auf ihre Verhaltensweisen und Routinen einwirken. Eine Analyse dieser Maßnahmen macht sichtbar, dass das betreute Wohnen weit mehr ist als eine Unterkunft oder ein „Dach über dem Kopf“". Es ist ein Raum des „Proto-Wohnens“ (Hasse, 2012:481). Seine Existenz legitimiert sich über das Versprechen, die in ihm Untergebrachten in den vermeintlich allgemeinen Funktionserfordernissen des Wohnens selbst zu unterweisen. Die „betreute Wohnung“ ist damit weniger Rückzugsort als Exerzierplatz und Modellwohnen. Wohnen ist hier nichts Selbstverständliches, Gewohntes und Gewöhnliches mehr - es ist zu einem Problem geworden, über das ständig reflektiert wird. Es ist ein Gegenstand der Problematisierung, an dem Regierungstechniken ansetzen (Foucault, 2005a:732). Deshalb ist ein Blick auf das betreute Wohnen und die hier wirksam werdenden Problematisierungen des unsicher gewordenen Wohnens heuristisch so aufschlussreich: Die Akteure der Wohnungslosenhilfe müssen Praktiken und Voraussetzungen des Wohnens, die als habitualisierte Selbstverständlichkeiten zumeist stumm bleiben, zur Sprache bringen. Die Analyse der Problematisierungen kann so die impliziten Normen des Wohnens in unserer Gesellschaft sichtbar und damit überhaupt erst kritisierbar machen. Mit Foucault können die vielen kleinteiligen Maßnahmen, die im betreuten Wohnen als Antwort auf das Problem der Wohnungslosigkeit zum Einsatz kommen, als politische Techniken verstanden werden. Es handelt sich um Mikrotechniken der Herstellung von Milieus, die Subjektivierungsprozesse in die gewünschte Richtung lenken sollen. Sie definieren gesellschaftliche Anforderungen an das Wohnen, die in Räumen der Hilfe institutionell fixiert werden und deren Verinnerlichung so zu einer sozialbehördlich verregelten und sozialarbeiterisch vermittelten Bedingung für die Integration von „Menschen in besonderen sozialen Schwierigkeiten“ (§67ff. SGB XII) wird.

Auffällig ist, dass etliche dieser Maßnahmen die Affekte und Gefühle der Wohnungslosen, ihre Bindung an die Wohnung, an Dinge in der Wohnung, an Haustiere und an andere Menschen adressieren. Das betreute Wohnen zeigt sich als äußerst aufmerksam für die emotionalen Akzente des Wohnens und für die Wohnung als Ort der gefühlsmäBigen Bewältigung gesellschaftlicher Erfordernisse. Inwieweit diese Bewältigung gelingen kann, ist in den Augen der Expert_innen vor allem eine Frage der Wohnfähigkeit. In 
der Hilfepraxis fungiert das Konzept der Wohnfähigkeit als selbstverständliche, sinnstiftende Verdichtung unterschiedlichster Aspekte, die im betreuten Wohnen zum Gegenstand fürsorglicher Einflussnahme werden. Das Konzept erzeugt institutionelle Signifikanz, indem es die Wissensproduktion über Wohnungslose anleitet; Einschätzungen über Wohnfähigkeit finden in Gutachten Niederschlag, die wiederum Entscheidungen über die Bewilligung und die Dauer von Hilfen für Wohnungslose informieren. Während der Betreuung werden verschiedenste Maßnahmen mit Verweis auf die Zielsetzung einer Steigerung der Wohnfähigkeit zum Einsatz gebracht.

Seiner institutionellen Bedeutung zum Trotz handelt es sich bei der Wohnfähigkeit aber auch um ein vages Konzept. Etliche Alltagshandlungen können im betreuten Wohnen zu Anhaltspunkten für mangelnde Wohnfähigkeit und damit zum Gegenstand regulativer Intervention werden: Die Beziehung der Wohnungslosen zu Geld und Zeit (1), ihr ,,Gefühl für sich selbst“ und den eigenen Körper (2), ihre emotionale Bindung an die Wohnung und an Dinge des alltäglichen Gebrauchs (3) sowie die Beziehungen zu anderen Menschen (4).

1. Mit seinem Fokus auf den Umgang von Wohnungslosen mit Geld und Zeit nimmt das betreute Wohnen Wohnungslosigkeit als Armutsphänomen in den Blick und macht das Wohnen zum Schauplatz der Bewältigung sozioökonomischer Exklusion. In vielen Interviews betonten die Expert_innen, dass Wohnungslosigkeit vor allem auf Arbeitslosigkeit, die Entwicklung der Wohnungsmärkte, die Sozialhilfereformen und die zudem rigide Sanktionierungspraxis der Jobcenter zurückzuführen sei. Eine neue Qualität von Verarmungsprozessen führe dazu, dass vielen Menschen objektiv zu wenig Geld zur Absicherung ihres Wohnraums zur Verfügung steht. Dennoch steht im Kontext der Hilfemaßnahmen de facto vor allem die Subjektivität der Betroffenen im Zentrum von Regulationsbemühungen. Die Expert_innen betonten auch, dass es vor allem auf den ,richtigen Umgang“ mit Geld ankomme, also darauf, Wohnungslosen eine vorausschauend kalkulierende Haushaltsführung beizubringen. Nicht ein objektiver Mangel an finanziellen Ressourcen, sondern die ökonomische Selbstführung rückt damit in den Mittelpunkt der Aufmerksamkeit.

In diesen auf die Selbstführung der Wohnungslosen abzielenden Problematisierungen werden rationale und emotionale Aspekte aufs Engste verknüpft. Vor allem der als affektgeleitet gedeutete Umgang von Wohnungslosen mit finanziellen Ressourcen ist Gegenstand edukativer Sorge. Viele Wohnungslose stünden ,,vor Schulden wie das Kaninchen vor der Schlange“, ein irrationaler „Tunnelblick“ verhindere eine vernünftige Auseinandersetzung mit der eigenen finanziellen Situation. Neben Entschuldungsplänen für die Klient_innen sind
Geldeinteilungssysteme eine wichtige Maßnahme, um Wohnungslose anzuleiten, ,auch mit kleinen Summen verantwortungsvoll umzugehen“. Diese Aktivierung der Eigenverantwortung zielt auf die Herausbildung eines kalkulativen Selbstmanagements ab, ein - wie die governmentality studies gezeigt haben (Procacci, 2000; Dean, 1992, 1995) - zentrales Element der Regierung von Armen, das diese ,ermächtigen“ soll, ,sich selbst zu helfen“ (Cruikshank, 1999).

Es darf aber nicht übersehen werden, dass im Zentrum der Bemühungen letztlich die Stärkung eines dieser Rationalität vorausgehenden Wollens, eine Kultivierung von ,economic sentiments“ (Rothschild, 2001) steht. Es geht um die Verbindung von „Kalkül und Leidenschaft“ (Vogl, 2004), denn es gilt, die richtige affektive Disposition gegenüber den finanziellen Aspekten des Lebens zu entwickeln. Wohnen ist immer auch Haushalten und Ökonomie ist nicht nur etymologisch (vom griechischen oikos) im Haushalt fundiert. Das richtige „Gefühl für Geld“ zu entwickeln, gilt deshalb als wesentliche Bedingung für gesellschaftlich gelungenes Wohnen.

Eng verbunden mit der Problematisierung des richtigen Umgangs mit Geld ist eine fürsorgliche Aufmerksamkeit für den Umgang von Wohnungslosen mit Zeit und Terminen. Der Alltag von Wohnungslosen ist durchdrungen von bürokratischen Erfordernissen und Behördengängen. In einigen Wohnprojekten führen Sozialarbeiter_innen in Posteingangslisten Buch über die Korrespondenz der Wohnungslosen mit Behörden wie dem Jobcenter. Regelmäßige Treffen von Sozialarbeiter_innen und Wohnungslosen sollen nicht zuletzt auch die Grundbereitschaft der Klient_innen steigern, Termine als normal hinzunehmen. Diese „Compliance“, also die Regelkonformität der Klient_innen, gilt als wesentliche Voraussetzung für Wohnfähigkeit. Wohnungslose werden darauf eingeschworen, sich in der Auseinandersetzung mit Behörden auf keinen Fall eigensinnig zu verhalten, da Nichtkooperation immer finanzielle Folgen haben kann, die das Wohnen erneut prekär werden lassen.

In diesen Versuchen, Wohnungslose im richtigen Umgang mit dem Jobcenter und anderen Behörden anzuleiten, bricht sich das „Aktivierungsparadigma“ (Lessenich, 2003). Das Regieren der Armen erschöpft sich eben nicht nur in Anrufungen zur Selbstermächtigung. Wohnfähig sein umfasst den Problematisierungen zufolge zwar, den eigenen Alltag ,eigenverantwortlich“ $\mathrm{zu}$ gestalten. Gleichzeitig wird Wohnungslosen aber auch die Akzeptanz und passive Erduldung von Erfahrungen der Fremdbestimmung und (mitunter abwegigen) bürokratischen Anforderungen nahegelegt.

2. Neben Versuchen der affektiven Strukturierung des Umgangs der Klient_innen mit Geld und Zeit bringt das be- 
treute Wohnen weitere Problematisierungen hervor, die den Zusammenhang von Wohnen und Gefühlen noch konsequenter ins Zentrum rücken. Mit seinem Fokus auf das „Gefühl [der Klient_innen] für sich selbst“, definiert das betreute Wohnen die Wohnung als Raum der Schonung und Selbstbewahrung. Da Wohnungslosigkeit immer wieder dazu führe, dass ,sich Leute auch selbst aufgeben“, ist die Anregung zur Selbstsorge ein explizites Ziel der Betreuung. Gelungene Selbstsorge wurde in den Interviews immer wieder als eine Frage von Sauberkeit und Körperhygiene konkretisiert: „Reinlichkeit, auf sowas gucken wir natürlich auch." Hygiene ist zentraler Gradmesser für ein gelungenes Selbstverhältnis: „Eine Wohnung sauber zu halten oder auch Körperpflege. Ich denke, es muss so eine, so eine Basis an Selbständigkeit muss einfach da sein. Sowohl was, was Körperpflege betrifft, aber auch eben Ämtergänge“.

3. Die Aufmerksamkeit für Selbstsorge adressiert neben der Körperpflege auch die Frage nach dem subjektiven Befinden im Wohnraum. Mit dieser Aufmerksamkeit für das Wohnerleben seiner Bewohner_innen thematisiert das betreute Wohnen die Wohnung als Ort der Behaglichkeit und emotionalen Bedürfniserfüllung. Bei der Suche nach überprüfbaren Indizien für häusliches Wohlbefinden gerät vor allem der Umgang der Wohnungslosen mit dem Inventar der Projektwohnung, mit Möbeln und Dingen des alltäglichen Gebrauchs in den Blick. „Die Wohnung schön machen“ gilt als Indiz für sinnvolles intimes Wohnverhalten, einen gelungenen Rückzug ins Innere (der den Interviews zufolge wohnungslosen Frauen oft besser gelinge als Männern). Gleichwohl wird aber auch davon ausgegangen, dass es so etwas wie ein richtiges Maß der Bindung an die Wohnung gibt. Wohnungslose sollen sich den Wohnraum zwar aneignen, sie dürfen dabei aber auch nicht zu weit gehen. Exzessives ,horden“ zeugt den Problematisierungen der Expert_innen zufolge von einer übersteigerten affektiven Bindung an Dinge, die erfolgreiches Wohnen unmöglich mache. „Wenn jemand nicht in der der Lage ist, sich von Dingen zu trennen, ständig neues Zeug anschleppt“", dann steht damit auch die Wohnfähigkeit in Frage. Die „Aneignung“ der Wohnung wird begrüßt, insofern sie zeigt, dass die Klient_innen die Wohnungen als etwas ihnen Zugehöriges betrachten. Gleichzeitig wird davon ausgegangen, dass sich hinter dieser „Aneignung“ potenziell auch zwanghaftes Verhalten verbergen kann. Erfolgreiches Wohnen umfasse schließlich auch, zu ,verstehen, was Müll ist“" ,Ich frag dann immer: ,Mal ehrlich, wofür brauchst Du das?““ Die Leitvorgabe einer ausbalancierten, ,gesunden“ Bindung bleibt in diesen Auseinandersetzungen zwangsläufig diffus: Wem die Herstellung einer ,gemütlichen“ Wohnatmosphäre gelingt oder was legitime Erweiterun- gen des Inventars sind, entscheiden letztlich die Sozialarbeiter_innen.

4. Eine ähnliche Gradwanderung vollzieht das betreute Wohnen in seiner Problematisierung akzeptabler Sozialität. Fürsorgliche Skepsis kann gegenüber starker Zurückgezogenheit, aber auch gegenüber ausgeprägter Geselligkeit entstehen. Einerseits gehöre es den Expert_innen zufolge zwar zum Wohnen dazu, Gäste zu empfangen und eventuell auch Beziehungen zum Wohnumfeld aufzubauen. Der Besuch von Gästen gilt als Indiz für eine erfolgreiche emotionale Raumnahme wie auch für die ,soziale Attraktivität" von Klient_innen. Zur Wohnfähigkeit gehöre es andererseits aber auch, Phasen des Alleinseins in der Wohnung auszuhalten. Erfolgreiches Wohnen umfasse die Fähigkeit, ,auch mal allein zu sein, ohne gleich am Rad zu drehen". Besuch kann insofern auch zu einem Indiz für eingeschränkte Wohnfähigkeit werden: „Es gibt ja welche, denen fällt die Decke auf den Kopf, sobald die allein sind, dann gehen die immer raus, oder holen sich alle Freunde rein. Das geht natürlich nicht." Auch die Frage, um wen es sich bei den Gästen handelt, kann im betreuten Wohnen bedeutungsvoll werden. $\mathrm{Zu}$ erfolgreichem Wohnen gehöre es auch, sich nicht länger ,auf der Straße zuhause [zu] fühlen" und eine emotionale Distanz zu Beziehungen aufzubauen, die sich dort möglicherweise entwickelt haben. Hierzu kann auch ein anderer Umgang mit Tieren gehören. Hunde etwa, die ein Leben auf der Straße gewöhnt waren und zu denen ihre Besitzer_innen oft intensive emotionale Bindungen aufgebaut haben, müssen im betreuten Wohnen ebenfalls domestiziert werden: ,die müssen auch erst lernen sich mehr wie richtige Haustiere zu benehmen“.

Eine Maßnahme, die in einigen betreuen Wohnprojekten zum Einsatz kommt, um das Alleinsein als Teil der Wohnfähigkeit zu trainieren, ist die Kontrolle von Besuchen: „Besuch, solange das geht, mal, ein zweimal in der Woche oder so [...] Aber immer nur in Absprache." Gleichwohl gehört es notwendig auch zum betreuten Wohnen, den regelmäßigen Besuch der Sozialarbeiter_innen zu akzeptieren, die sich in einigen Wohnprojekten mit eigenen Schlüsseln auch unangekündigt $\mathrm{Zu}$ gang zu verschaffen, um den Zustand der Wohnung in Augenschein zu nehmen.

Insgesamt gilt die Arbeit an der eigenen Selbstführung im betreuten Wohnen als entscheidende Voraussetzung für die Reinklusion in gesellschaftliche Zusammenhänge. Diese Arbeit an der eigenen Selbstführung erweist sich im betreuten Wohnen auch als eine Arbeit an Gefühlen. Die Hilfe ist durchzogen von Problematisierungen, die ausbalancierte Gefühlszustände zum Ziel erklären und dabei immer wieder normalisierende Anforderungen formulieren: Es gilt, nicht zuwenig, aber auch nicht zuviel Bindung an die Wohnung, 
ihr Mobiliar, Dinge des alltäglichen Gebrauchs, Haustiere und andere Menschen zu entwickeln.

\section{Wohnfähigkeit anders problematisieren: Fluchtlinien der Kritik}

Mit Foucaults Analytik der Gouvernementalität kann die Wohnfähigkeit als ein Konzept interpretiert werden, dass die auf Wohnungslosigkeit bezogene Regierungspraxis begrifflich fasst. Es macht eine „Rationalisierung der Regierungspraxis“ möglich, indem es „Anwendungsfelder“ konstituiert (Räume des betreuten Wohnens), „Gegenstände und Regeln“ des Regierens bestimmt (wohnungslose Subjekte, ihr Verhalten und ihre Kooperationsbereitschaft), ,aufs Ganze gerichtete Ziele" verfolgt (die gesellschaftliche Re-Integration der Wohnungslosen) und zum Erreichen dieser Ziele die „bestmögliche Weise zu regieren“ vorschlägt (Arbeit an der defizitären wohnungslosen Subjektivität als Mittel zur Realisierung gesellschaftlicher Re-Inklusion) (Foucault, 2004:14).

Ähnlich wie die sogenannte employability bzw. „Beschäftigungsfähigkeit", an der es lebenslang zu arbeiten gilt, um legitimen Anspruch auf die Inklusion in Arbeitsverhältnisse geltend machen zu können, erklärt auch die Regierung der Wohnungslosigkeit mit dem Konzept der Wohnfähigkeit die Arbeit an der eigenen Subjektivität zur Voraussetzung für die Teilhabe am Wohnen und stellt Trainingsräume bereit, in denen sich die Subjekte beweisen sollen. Eine Analyse von Problematisierungen kann diese gesellschaftliche Antwort auf Wohnungslosigkeit als eine therapeutischedukative Form des Regierens sichtbar machen, die sich ganz auf das einzelne Subjekt kapriziert. Das betreute Wohnen wird sichtbar als eine „Heterotopie“, die gesellschaftliche Widersprüche prozessiert - nicht zuletzt auch, indem sie sie versteckt (Foucault, 2005b, c). Strukturelle Ursachen, die einer Überwindung von Wohnungslosigkeit im Weg stehen (angespannte Wohnungsmärkte, Arbeitslosigkeit und niedrige Grundsicherungsleistungen) kann diese Form der Hilfe nicht adressieren, Folgeeffekte von Armut und Verschuldung, die den Wiedereinstieg in den regulären Mietmarkt verhindern (SCHUFA-Einträge und fehlende Mitschuldenfreiheitsbescheinigungen), kann sie kaum beseitigen. Angesichts dieser Limitierungen verwundert es nicht, dass Einschätzungen über Erfolge des Hilfesystems häufig selbst referentielle Argumentationslinien einschlagen. Ein erfolgreiches Betreuungsverhältnis wird dann nicht unbedingt am Bezug einer eigenen Wohnung durch die Klient_innen festgemacht, sondern eher prozessual daran, ob sich ihr Wohnverhalten während der Betreuung verändert. Unzufriedenheit von Wohnungslosen mit dem Hilfesystem oder „gescheiterte Fälle" werden mitunter dahingehend interpretiert, dass noch „passgenauere Hilfen“, also eine noch intensivere Auseinandersetzung mit dem/der Einzelnen und noch engere $\mathrm{Zu}-$ sammenarbeit der Instanzen des Hilfesystems nötig seien. Im Anschluss an Foucault haben Hubert Dreyfus und Paul Ra- binow (1983:196) diese immunisierende Logik von Expertenwissen treffend auf den Punkt gebracht: „Where there [is] resistance, or failure to achieve its stated claims, this [is] construed as further proof of the need to reinforce and extend the power of experts [...]. When they fail, this only justifies the need for more of the same."

Es liegt nahe, auf diese Widersprüche mit einer Sozialkritik zu reagieren, die an den strukturellen Gründen für Wohnungsverluste ansetzt, um diese Gründe - und nicht den einzelnen Wohnungslosen - ins Zentrum der Aufmerksamkeit zu rücken. Diese Form der Kritik ist sicher richtig, was sie allerdings aus dem Blick geraten lässt, ist eine kritische Auseinandersetzung mit dem operativen Konzept der Wohnfähigkeit selbst und mit dem Verständnis von Wohnen, das es produziert. Für Foucault haben Problematisierungen immer eine Doppelfunktion, sie sind Untersuchungsgegenstand und kritisches Instrument zugleich und funktionieren damit auf der Ebene der Beobachtung erster und zweiter Ordnung. Aufgabe einer kritischen Analyse ist es demnach, Problematisierungen zu problematisieren. Im Hinblick auf Wohnfähigkeit wäre dann zu fragen, ob es sich beim Wohnen überhaupt um eine subjektiv unterschiedlich stark ausgeprägte „Fähigkeit“" handelt und welche Effekte dieses Verständnis von Wohnen als eine im einzelnen Individuum angelegte, isolierbare Fähigkeit im Hilfesystem nach sich zieht. Kann die betreute Projektwohnung überhaupt ein „Zuhause“ werden, oder verhindern die etablierten administrativen Formen des behördlichen Leistungstyps „,betreutes Wohnen" nicht viel eher, dass die als hilfebedürftig Identifizierten in den Projektwohnungen tatsächlich wohnen können? Welche Möglichkeiten es gibt, eine solche Reproblematisierung des Regierens der Wohnungslosigkeit zu entwickeln, die die Expert_innen beim Wort nimmt, indem sie am Konzept der Wohnfähigkeit selbst ansetzt, soll im folgenden Kapitel ausgelotet werden, das sich dem Wohnen mithilfe von nicht-subjektzentrierten und affekttheoretischen Theorien annähert.

\section{Dem Wohnen auf der Spur}

Philosophien des Wohnens eröffnen unterschiedliche Möglichkeiten, die diffusen gesellschaftlichen und behördlichen Vorstellungen von Wohnen zu hinterfragen, die in der Praxis des betreuten Wohnens ihre Wirksamkeit entfalten. Eine bedeutsame philosophische Bestimmung von Wohnen findet sich im Werk Martin Heideggers. Wohnen wird hier als existentieller Ausdrucksbereich individuellen wie auch gesellschaftlichen Lebens verstanden. Für Heidegger ist Wohnen nichts weniger als ,die Art, wie du bist und ich bin, die Weise, nach der wir Menschen auf der Erde sind [...]. Mensch sein heißt: als Sterblicher auf der Erde sein, heißt: wohnen" (2004:141, Hervorhebung i.O.). Eine solche Bestimmung des Wohnens überschreitet Fragen nach dem „Verortet-sein“ an einem Wohnort oder dem „Eingerichtet-sein“ in einer Woh- 
nung und zielt auf den Zusammenhang von Wohnen und Leben, auf den tatsächlichen, leiblichen und politischen Raum der Lebensführung ab. So verstanden ist Wohnen aber gerade nichts Erratisches und aus konkreten Lebensvollzügen Isolierbares, wie es die Hilfepraxis für Wohnungslose unterstellt. Das wohnende Subjekt ist nicht einfach nur an einem Wohnort lokalisierbar und dort von der Wohnung und ihren Funktionen umgeben. Wohnen verstanden als existenzieller Ausdruck des Lebens muss nicht auf den Ort der Wohnung beschränkt sein und ist streng genommen auch kein rationalistisches Handeln, das auf messbare, unterschiedlich stark ausgeprägte Fähigkeiten zurückführbar wäre. Über den Raum des Wohnens realisieren sich vielmehr die praktisch gelebten Verhältnisse zur Welt wie auch zum Selbst. Das wohnende Subjekt existiert, indem es ekstatisch ,außer-sich“ bei sich selbst zu Hause ist.

Von Wohnfähigkeit zu sprechen, erscheint vor dem Hintergrund einer solchen Bestimmung von Wohnen einigermaßen paradox. Die vielen Mikropraktiken, die im betreuten Wohnen zur Steigerung der Wohnfähigkeit beitragen sollen, arbeiten auf die Erzeugung rationalistischer, kompetenter Akteure hin, die sich ihrer selbst allzeit bewusst sind. In der Hilfe für Wohnungslose wird Wohnen ausgelegt als Vermögen, eine aktive Potenz, die sich trainieren lässt; Wohnen steht und fällt mit dem einzelnen Subjekt und seiner Leistungsfähigkeit. Das Konzept der Wohnfähigkeit impliziert individuelle Selbstbeherrschung im Angesicht gesellschaftlicher Anforderungen und die Bereitschaft, sich dem als ,normal“ imaginierten Wohnen möglichst weit anzunähern. Damit räumt das betreute Wohnen seinen Klient_innen aber gerade keine ausreichenden Möglichkeiten ein, ihre Lebenssituation im Sinne Heideggers ,im Spiegel des Wohnens zum Ausdruck“ (Hasse, 2009:19, Hervorhebung i.O.) zu bringen und damit Macht über ihr Leben (zurück) zu gewinnen.

Wenn Wohnen bestimmt wird als eine auf das ganze Leben bezogene Form der Selbstentfaltung, eine Seinsweise mit spezifischen Voraussetzungen, dann stellt sich die Frage, ob diese Voraussetzungen im „,betreuten Wohnen“ erfüllt sind. Konkret wäre der Frage nachzugehen, welche leiblichgefühlsmäßigen Dispositionen das betreute Wohnen erzeugt. Vor diesem Hintergrund wird auch die Politik der Gefühle fragwürdig, die die Praxis der Betreuung auszeichnet. Phänomenologische Theorien des Wohnens betonen den Zusammenhang zwischen Raum und leiblicher Befindlichkeit und damit die Bedeutung der atmosphärischen Wirkung von Räumen (Böhme, 2006:122). Bei Hermann Schmitz (1977:213) findet sich eine Bestimmung von Wohnen als ,,Verfügen über Atmosphärisches“. Werden einem solchen Verfügen im Kontext des betreuten Wohnens Spielräume gewährt? Die atmosphärische Wirkung der Betreuungssituation und ihre Effekte auf die Klient_innen werden in der Praxis kaum systematisch reflektiert. Gleichwohl ist davon auszugehen, dass das Ziel der Erzeugung rationalistischer, kompetenter Wohn-Akteure eine Grundstimmung im betreuten Wohnen erzeugt, die der Möglichkeit atmosphärischer Geborgenheit als Kennzeichen von Wohnen eher entgegenarbeitet, als $\mathrm{zu}$ ihr beizutragen. Geborgenheit entsteht schließlich vor allem in solchen Kontexten, in denen nicht permanent Leistungsfähigkeit unter Beweis gestellt werden muss. Gelungenes Wohnen müsste einer „entlastenden Seinsweise“ (Hasse, 2009:14) Spielraum geben, die gerade nicht von autoritativer Wissensproduktion und engen Vorgaben für legitime Raumaneignung durchdrungen ist, sondern die Beziehung zwischen Innen und Außen als eine „Kultivierung umfriedeter Atmosphären“ (ebd.: 28) gestaltet. Bedingung für Wohnen ist, dass es als Ausgleichswelt funktioniert und damit eine „Sorge um sich“ (Foucault, 1998) ermöglicht, über die die Wohnenden selbst verfügen (Schmitz, 1977:213). Eine Selbstsorge, die zunächst keiner äußerlichen Zielsetzung folgt und ganz in gesellschaftlichen Begründungen aufgeht.

Zur Evaluation und Steigerung der Leistungsfähigkeit des wohnungslosen Subjekts schafft das betreute Wohnen eine räumliche Laborsituation. Die Projektwohnung wird zum Reagenzglas, in dem der/die Einzelne sich beweisen und an Zielvereinbarungen messen lassen muss. Damit werden im betreuten Wohnen spezifische Sichtbarkeitsordnungen Formen von sehen und gesehen-werden und eingeschränkte Möglichkeiten, sich den Blicken anderer zu entziehen gestiftet. Von Expert_innen wird das betreute Wohnen vor allem geschätzt, weil es „normalem Wohnen“ ähnlicher sei als große Notunterkünfte. Dies trifft für die einzelne Projektwohnung im Sinne einer „Behälterform“ zwar zu, weit weniger aber für die sozialen Beziehungen und Sichtbarkeiten, die das betreute Wohnen erzeugt, wenn es das Wohnen als verborgenen, sensiblen Vorgang ins Licht pädagogischer Aufmerksamkeit rückt. Es etabliert dabei eigene Modi von Innen und Außen, Öffnen und Schließen, die es vom „normalen Wohnen“"wesentlich unterscheiden. Die black box unterschiedlichster Alltagsvorgänge wird einem kontrollierenden Blick und erzieherischen Eingriffen zugänglich gemacht: Das betreute Wohnen führt Buch über die Post der Klient_innen, es macht sich ein Bild über Termine und Kommunikation seiner Bewohner_innen mit Behörden, es beleuchtet den Umgang der Bewohner_innen mit sich selbst und mit der Wohnung und beobachtet ihre Bindungen an Dinge und andere Menschen, zu diesem Zweck öffnen Sozialarbeiter_innen die Projektwohnungen unter Umständen auch mit eigenen Schlüsseln. Damit ist das betreute Wohnen ein „Ort konstruierter Sichtbarkeit“ (Rajchman, 2000:51), durchzogen von Strategien der Wissensproduktion. Wohnungslose werden immer wieder aufgefordert, Einblick in ihre Wohnungen und Auskunft über sich selbst zu geben.

Auch Philosophien, die anders als Heidegger und an ihn anschließende phänomenologische Ansätze vor allem den Ortsbezug des Wohnens in den Mittelpunkt des Interesses rücken, ermöglichen einen veränderten Blick auf diese Praxis des betreuten Wohnens und die Justierung von Gefühlen, die in den Projektwohnungen realisiert werden soll. Für Emmanuel Levinas etwa ist die Wohnung ein „Apparat der für den Menschen lebensnotwendigen Dinge“ (Levinas, 1987:217). 
Levinas schreibt über das Wohnen: „Die eigentliche Weise, sich zu halten, ist das Wohnen [...] Das ,Bei-sich-zuHause' [le ,chez-soi'] ist kein Behälter, sondern ein Ort, an dem ich kann, an dem ich, obwohl abhängend von einer anderen Realität, frei bin. Der Ort, die Mitte [le milieu], hält Mittel und Wege bereit. Alles ist hier, alles gehört mir, alles ist be-griffen.“ (1987:42, Betonung i.O.) Das „Bei-sich-zuHause" ist für Levinas vor allem emotionaler Schonraum und Rückzugsort und damit ein Ort der relativen Autonomie. Teil dieser Autonomie und der Möglichkeiten zu Rückzug und Selbstschonung ist auch der Zeithorizont, den das Wohnen eröffnet. Das betreute Wohnen ist geprägt von Kurzfristigkeit und Vorläufigkeit. Wohnungslose sollen häusliches Wohlbefinden erleben und die Möglichkeit erhalten, emotional ,zur Ruhe zu kommen“, um die Stresssituation der Wohnungslosigkeit zu verwinden. Die Projekte können gleichwohl immer nur befristetes Wohnen bereitstellen. Es gilt, sich zuhause zu fühlen, aber auch zu, ,akzeptieren, dass das eben nichts für immer ist“. Das Wohnen qualifiziert als „Bleibe“ (Levinas, 1987:215) müsste demgegenüber aber eigentlich auch ein Ort sein, der (zumindest eingeschränkte) Möglichkeiten des Bleibens eröffnet und selbstbestimmte Modi von Kommen und Gehen erlaubt. Dass „Leute [aus den Projektwohnungen] manchmal gar nicht mehr rauswollen, aber rausmüssen“ erzählt von emotionalen Verletzungen, die das Hilfesystem trotz aller guten Absichten selbst produziert.

Verbunden mit der Vorläufigkeit des betreuten Wohnens ist die normalisierende Anforderung, sich an die Projektwohnung zu binden, aber auch nicht zu sehr an ihr zu hängen. Es geht in diesem (neoliberalen) Regieren von Gefühlen offenbar darum, flexibel zu bleiben, sein Herz nicht zu sehr an Dinge und Orte zu hängen, zu lernen, dass ,nichts für immer" ist. Aus affekttheoretischer Perspektive sind solche Anrufungen einigermaßen paradox. Nicht nur ist fragwürdig, $\mathrm{ob}$ es ein erstrebenswertes Ziel ist, emotionale Bindungen auf diese Weise zu regulieren, es ist auch fraglich, ob dies überhaupt möglich ist - ob wir in der Lage sind, unsere Bindungen gänzlich autonom zu gestalten und zu normalisieren, oder ob die Orte und Dinge die uns affizieren, dabei nicht immer auch ein Wort mitzureden haben. Als transsubjektives Wechselspiel von affizieren und affiziert-werden hängt gelungenes Wohnen elementar von unserer sinnlichen Kommunikation mit Dingen ab (Guzzoni, 2008). „Die Wohnung ist wie ein Nest, in das man Dinge trägt, mit denen man leben will." (Hasse, 2009) Als ein solcher Ort ist die Wohnung auch ein Freiheitsfeld für Idiosynkrasien. Im Sinne rationalistischer Perspektiven unterstellt das betreute Wohnen, dass kompetente Subjekte jederzeit schlüssig Auskunft darüber geben können (und müssen), warum sie sich mit welchen Dingen umgeben (,Ich frag dann immer: ,Mal ehrlich, wofür brauchst du das?"“). Im geglückten Wohnen besteht affekttheoretischen Perspektiven zufolge aber gerade kein Rechtfertigungsdruck darüber, warum uns Dinge glücklich machen und mit welchen Dingen wir uns umgeben möchten. Jane Bennett, eine Vertreterin des new materialism, verweist im Hinblick auf sogenannte „Horder“ und „Messies“ darauf, dass es natürlich leicht ist, das exzessive Sammeln von Dingen als Zwangsstörung, als nachgerade typische Psychopathologie des Konsumkapitalismus zu deuten. Vielleicht wäre es Bennett zufolge aber viel interessanter, darüber nachzudenken, was uns hording über den Reiz, den Dinge auf uns ausüben, erzählen kann, über die Art, wie sie zu uns sprechen: ,There are non-linguistic communications at work between vibrant materials, and if they are there, maybe we can try to develop a vocabulary for describing them and cultivating a sensitivity to them" (Bennett, 2011). Sara Ahmed stellt fest, dass wir über dieses Vokabular teilweise bereits verfügen, etwa wenn wir sagen, dass uns etwas glücklich macht und damit die affektive Macht von Dingen adressieren: „To be ,made happy ' by this or that is to recognize that happiness starts from somewhere other than the subject" (Ahmed, 2010:29). Auch die Normalisierung der Sozialität im betreuten Wohnen, die Beobachtung von Besuchen und die Aufforderung, das Alleinsein in der Projektwohnung auszuhalten, erscheint aus der Perspektive einer Theorie des Wohnens fraglich, für die das Bei-sich-sein die Begegnung mit dem Anderen immer schon impliziert: „bei sich zu Hause sein [heißt], daß man zu Hause empfängt, dorthin einlädt, dort seine Gastfreundschaft anbietet, sich also einen Ort aneignet, um den Anderen dort zu empfangen oder, schlimmer noch, daß man den Anderen dort empfängt, um sich einen Ort anzueignen und die Sprache der Gastfreundschaft zu sprechen“"(Derrida, 1999:33, Betonung i.O.). Wenn Wohnen aber heißt, den Anderen einzuladen, auf ,das Klopfen des Anderen an der Tür zu antworten“, dann etablieren Kontrollbesuche und der unangekündigte Eintritt von Sozialarbeiter_innen in die Projektwohnungen eine Politik der Türschwelle, die genau die Autonomie verletzt, die Voraussetzung für eine gelungene Begegnung mit dem Anderen im Wohnen wäre.

\section{Fazit}

Der Umgang mit Wohnungslosen im betreuten Wohnen ist unter Expert_innen der Wohnungslosenhilfe nicht unumstritten. Mehrfach wurden in den Interviews distanzierte Positionen zum Konzept der Wohnfähigkeit eingenommen, etwa in Form der ironisierenden Einschätzung, man könne ,natürlich jeden irgendwie für nicht wohnfähig erklären, wenn man das will“". Einzelne Sozialarbeiter_innen betonten, dass sie von den zur Verfügung stehenden Kontrollspielräumen nicht vollständig Gebrauch machen: „Andere machen das, klar, aber wir gehen nicht einfach in die Wohnungen. Wir klingeln lieber erst zehnmal [...] Wir schließen nur auf, wenn wir uns echt Sorgen machen. Normalität heißt ja auch, dass da nicht ständig wer rein spaziert.“

Aber auch wenn es unterschiedliche Modi des Umgangs mit den Vorgaben gibt, so strukturiert das herausgearbeitete Verständnis vom Zusammenhang von Subjektivität und 
Wohnen doch das Feld des Möglichen der Wohnungslosenhilfe. Die hier wirksame Problematisierung der defizitären Subjektivität der Wohnungslosen stabilisiert sich zudem selbst, indem sie institutionelle „looping effects“ (Hacking, 2007, 286) erzeugt, die sich dem Zugriff einzelner Sozialarbeiter_innen entziehen: Damit Hilfen für Wohnungslose überhaupt bewilligt werden, muss ihre Wohnfähigkeit geradezu infrage gestellt werden, denn die „,besonderen sozialen Schwierigkeiten“, die nach $\$ 67 \mathrm{ff}$. Sozialgesetzbuch XII Voraussetzung der Hilfebewilligung sind, müssen schließlich auch vorliegen. Der schillernd vieldeutige Charakter des Konzepts der Wohnfähigkeit erweist sich als Vorteil angesichts dieses Beweisführungsdrucks, der auf den Hilfeinstanzen lastet.

Durch das Konzept der Wohnfähigkeit und die mit ihm einhergehende Hilfepraxis werden die Akteure der Wohnungslosenhilfe auf eine bestimmte „Ontologie“ des Wohnens und der Subjektivität verpflichtet, die nicht nur einem komplexeren Verständnis von Wohnen nicht gerecht wird, sondern zudem auch paradoxe Effekte zeitigt. Um dies zu zeigen wurde die „Ontologie“ des betreuten Wohnens mit alternativen Perspektiven auf Wohnen und Subjektivität kontrastiert. Diesen diversen Ansätzen aus Phänomenologie, Poststrukturalismus, Affekttheorie und New Materialism ist gemeinsam, dass sie Wohnen nicht als Fähigkeit eines Subjekts verstehen, sondern als eine Seinsform, die Personen, Dinge, Gefühle und Affekte, Beziehungen zwischen Menschen und Nicht-Menschen versammelt. Mithilfe dieser Ansätze wird Wohnen denkbar als eine Versammlung, ein „Gefüge“ (Deleuze und Guattari, 2002:194). Diese Gefüge enthalten zwar die Wohnenden und ihre Praktiken, doch sie gehen nicht in diesen Praktiken auf, sondern umfassen auch Beziehungen, Begehren, Dinge, Stimmungen, Atmosphären, Rhythmen und Resonanzen. Analytisch vom Wohnen als Versammlung aus zu denken, heißt nicht, dass die Wohnenden ganz aus dem Blick verschwänden. Aber während das Subjekt im betreuten Wohnen vorausgesetzt wird - es ist bereits da, hat defizitäre Fähigkeiten und wird dann in die Trainingswohnung gesetzt - ist es aus einer nicht-subjektzentrierten Perspektive selbst ein Effekt der Versammlung. „Subjektivierung [setzt] ein Gefüge voraus, und nicht umgekehrt" (ebd.: 193).

Aus dieser Perspektive wird der paradoxe Charakter des Wohnfähigkeitstrainings deutlich, das Wohnen als ein unterschiedlich stark ausgeprägtes subjektives Vermögen angeht. Es wird sichtbar, dass die Hilfepraxis mit ihren Auflagen und Kontrollen das Wohnen in einem anspruchsvollen Sinn oft eher verkompliziert, anstatt zu ihm zu befähigen. Die von Sozialarbeiter_innen gelegentlich problematisierte „,noncompliance“ ihrer Klient_innen ist - gemäß der im zweiten Teil des Aufsatzes entwickelten Lesart - vielleicht gerade kein Indiz für mangelnde Wohnfähigkeit, sondern auch ein Hinweis auf Momente emanzipatorischer Selbstbehauptung - Versuche einer „Sorge um sich“ (Foucault, 1998), die sich gegen die affektive Justierung von Lebensgefühlen im be- treuten Wohnen sträuben. Wohnen verstanden als „Sorge um sich“ geht nicht auf in selbstbezüglichen oder gar ,,neoliberalen" Selbstoptimierungspraktiken, sondern schließt immer schon einen Welt- bzw. Umweltbezug - Sorge um sich - mit ein. Beziehungen zur Dingwelt als auch Beziehungen zu anderen Menschen sind ebenso Teil dieser Sorge, wie der existenzielle Selbstbezug.

Wie könnte eine Hilfe für Wohnungslose aussehen, die ihre Klient_innen darin unterstützt, Macht über ihr eigenes Leben zu gewinnen? Eine Hilfe, die den affektiven, leiblichgefühlsmäßigen Dimensionen des Wohnens Spielraum gibt? Die Kontrastierung des betreuten Wohnens mit alternativen Perspektiven auf Wohnen und Subjektivität liefert keine handliche Richtschnur für ein gutes „Management“ von Wohnungslosigkeit. Sie liefert auch keine normativen Kategorien in dem Sinne, dass sie allgemeingültige Kriterien für „richtiges Wohnen“ aufstellen würde. Was sich aber aus diesen Theorieperspektiven ergibt, ist durchaus so etwas wie eine Ethik des Wohnens, ein Nachdenken, das keine substanziellen Vorgaben macht, sondern so etwas wie eine Vorbereitung oder Vorübung für gutes Wohnen ist.

Danksagung. Mein besonderer Dank gilt den beiden anonymen Gutachter_innen der Geographica Helvetica sowie Benedikt Korf als Editor für ihre ausgesprochen konstruktiven Anmerkungen und Vorschläge.

Edited by: B. Korf

Reviewed by: two anonymous referees

\section{Literatur}

Ahmed, S.: Happy Objects, in: The Affect Theory Reader, Herausgeber: Gregg, M. und Seigworth, G. J., Duke University Press, Durham, 29-51, 2010.

Barry, A., Osborne, T., und Rose, N., Herausgeber: Foucault and political reason: Liberalism, neo-liberalism, and rationalities of government, Routledge, Chicago, 1996.

Bennett, J.: Powers of the Hoard. Notes on Material Agency, Vortrag am ICI Berlin, Internet: https://www.ici-berlin.org/de/ videos/bennett/ (letzter Zugriff: 25 Mai 2014), 2011.

Böhme, G.: Architektur und Atmosphäre, München, Wilhelm Fink Verlag, 2006.

Braidotti, R.: Bio-Power and Necro-Politics, Internet: http: //www.springerin.at/dyn/heft_text.php?textid=1928\&lang=en (letzter Zugriff: 25 Mai 2014), 2007.

Bröckling, U.: Das unternehmerische Selbst: Soziologie einer Subjektivierungsform, Suhrkamp Verlag, Frankfurt/M., 2007.

Bröckling, U.: In der Optimierungsfalle. Zur Soziologie der Wettbewerbsgesellschaft, Supervision, Mensch Arbeit Organisation, 31, 4-11, 2013.

Cruikshank, B.: The will to empower: Democratic citizens and other subjects, Cornell University Press, Ithaca, 1999.

Dean, M.: A genealogy of the government of poverty, Econ. Soc., $21,215-251,1992$. 
Dean, M.: Governing the unemployed self in an active society, I. J. Hum. Resour. Man., 24, 559-583, 1995.

Deleuze, G. und Guattari, F.: Tausend Plateaus. Kapitalismus und Schizophrenie, Merve Verlag, Berlin, 2002.

Derrida, J.: Adieu. Nachruf auf Emmanuel Levinas, Carl Hanser Verlag, München, 1999.

Dreyfus, H. L. und Rabinow, P.: Michel Foucault: Beyond Structuralism and Hermeneutics, University of Chicago Press, Chicago, 1983.

Foucault, M.: Die Sorge um sich. Sexualität und Wahrheit 3, Frankfurt/M., 1998.

Foucault, M.: Geschichte der Gouvernementalität II. Die Geburt der Biopolitik. Vorlesungen am Collège de France 1978-1979, Frankfurt/M., 2004.

Foucault, M.: Politik, Polemik und Problematisierungen, in: Michel Foucault. Dits et Ecrits. Schriften in vier Bänden. Band IV 19801988, Herausgeber: Defert, D. und Ewald, F., Suhrkamp Verlag, Suhrkamp Verlag, Frankfurt/M., 724-734, 2005 a.

Foucault, M.: Die Heterotopien. Der utopische Körper. Zwei Radiovorträge, Suhrkamp Verlag, Suhrkamp Verlag, Frankfurt/M., 2005b.

Foucault, M.: Von anderen Räumen, in: Michel Foucault. Dits et Ecrits. Schriften in vier Bänden. Band IV 1980-1988, Herausgeber: Defert, D. und Ewald, F., Suhrkamp Verlag, Frankfurt/M., 931-942, 2005c.

Guzzoni, U.: Unter anderem: die Dinge, Verlag Karl Alber, Freiburg, 2008.

Hacking, I.: Kinds of People: Moving Targets, P. Brit. Acad., 151, 285-318, 2007.

Hasse, J.: Unbedachtes Wohnen. Lebensformen an den verdeckten Rändern der Gesellschaft, transcript Verlag, Bielefeld, 2009.

Hasse, J.: Wohnen, in: Handbuch Stadtsoziologie, Herausgeber: Eckhardt, F., VS Verlag für Sozialwissenschaften, Wiesbaden, 475-502, 2012.
Heidegger, M.: Bauen Wohnen Denken, in: Vorträge und Aufsätze, Klett-Cotta, Stuttgart, 139-156, 2004.

Lemke, T., Krasmann, S. und Bröckling, U.: Gouvernementalität der Gegenwart. Studien zur Ökonomisierung des Sozialen, Suhrkamp Verlag, Frankfurt/M., 2000.

Lessenich, S.: Der Arme in der Aktivgesellschaft. Zum sozialen Sinn des Forderns und Forderns, WSI Mitteilungen, 56, 214 220, 2003.

Levinas, E.: Totalität und Unendlichkeit. Versuch über die Exteriorität, Verlag Karl Alber, Freiburg, München, 1987.

Procacci, G.: Poor Citizens. Social Citizenship versus Individualization of Welfare, in: Citizenship, markets, and the state, Herausgeber: Crouch, C., Eder, K., und Tambini, D., Oxford, 49-68, 2000.

Rajchman, J.: Foucaults Kunst des Sehens, in: Imagineering: visuelle Kultur und Politik der Sichtbarkeit, Herausgeber: Holert, T., Oktagon, Köln, 40-63, 2000.

Rose, N.: Inventing ourselves, Cambridge University Press, Cambridge, 1998.

Rose, N.: Powers of Freedom: reframing political thought, Cambridge University Press, Cambridge, 1999.

Rothschild, E.: Economic Sentiments. Adam Smith, Condorcet, and the Enlightenment, Harvard University Press, Harvard, 2001.

Schmitz, H.: System der Philosophie, Band III Der Raum, 4. Teil: Das Göttliche und der Raum, Bouvier Verlag, Bonn, 1977.

Specht, T.: Von der Wohnungslosenhilfe zu Hilfen zur sozialen Inklusion für Wohnungsnotfälle, Archiv für Wissenschaft und Praxis der sozialen Arbeit, 1, 4-21, 2013.

Stoler, A. L.: Affective States, in: A Companion to the Anthropology of Politics, Herausgeber: Nugent, D. und Vincent, J., WileyBlackwell, Oxford, 4-20, 2008.

Vogl, J.: Kalkül und Leidenschaft. Poetik des ökonomischen Menschen, diaphanes, Zürich, 2004. 